\title{
A Methodology for Designing Natural Language Interfaces for Procedural Content Generation
}

\author{
Afshin Mobramaein \\ University of California, Santa Cruz \\ Santa Cruz, CA, USA \\ mobramaein@soe.ucsc.edu
}

\author{
Jim Whitehead \\ University of California, Santa Cruz \\ Santa Cruz, CA, USA \\ ejw@soe.ucsc.edu
}

\begin{abstract}
Procedural Content Generation (PCG) uses algorithmic techniques to create a wide variety of content for games. These generators often have a large number of parameters, making it difficult for non-technical designers to explore the design space of generated artifacts. Natural language interfaces for generators can map natural language keywords to parameter space changes spanning multiple simultaneous parameters and afford use of expressive language. This way, designers can navigate to interesting points in the design space of a generator by describing desired properties of the artifact using a series of natural language descriptors. We present a design methodology that designers can use to develop natural language interfaces for procedural content generation systems. This design methodology begins by defining a design vocabulary that can describe the output of a generator, mapping the vocabulary to a series of parameters, and translating natural language queries to movements in the generator's design space. We further address issues around designer intent understanding, design space exploration and workflows using natural language interfaces in PCG. An example and implementation of our methodology is provided demonstrating its application to existing plug-ins for content creation in the Unity3D engine
\end{abstract}

\section{CCS CONCEPTS}

- General and reference $\rightarrow$ Design; - Human-centered computing $\rightarrow$ Interaction design theory, concepts and paradigms; $\bullet$ Software and its engineering $\rightarrow$ Interactive games;

\section{KEYWORDS}

Natural language interfaces; procedural content generation; design methodology

\section{Introduction and Motivation}

The use of procedural content generation (PCG) in games lets technical designers leverage the expressive power of algorithmic

Permission to make digital or hard copies of part or all of this work for personal or classroom use is granted without fee provided that copies are not made or distributed for profit or commercial advantage and that copies bear this notice and the full citation on the first page. Copyrights for third-party components of this work must be honored. Abstracting with credit is permitted. To copy otherwise, or republish, to post on servers or to redistribute to lists, requires prior specific permission and/or a fee. Request permissions from Permissions@acm.org.

FDG '19, August 26-30, 2019, San Luis Obispo, CA, USA

(C) 2019 Association for Computing Machinery.

ACM ISBN 978-1-4503-7217-6/19/08 _.\$15.00

https://doi.org/10.1145/3337722.3341860 techniques to create large amounts of a specific type of content. Some of the advantages of using PCG are reducing the amount of workload to create a large number of consistent assets or increasing the replay value of a game. Complex PCG systems typically have a high dimensional design space that is difficult to explore, and hence can be difficult to use and modify by nontechnical users. Understanding the interplay of the underlying parameter space and generative techniques is the key driver of the difficulty in using PCG system.

Mixed-initiative PCG systems try to ease the burden on users by allowing a cooperation between the procedural generator and a user. Such systems provide a simplified user interface that lets the user pass constraints to the generator in order to co-create an artifact. Even so, exposing a large series of inputs that control the generation of artifacts can prove a daunting task to users not familiar with the design space of these generative methods. One such example is Cillr [1,2] by Nelson et al., a PCG system that allows the creation of mobile games on-device. In Cillr, the user is exposed to a UI control for each dimension in the design space, 284 to be specific. Nelson et al. [1,2] mention that users interacting with the system during user testing had difficulty navigating the large amount of UI controls, and were sometimes frustrated when the manipulation of these controls would not conform to their expectations.

Another issue that arises is capturing the desired intent of the user via the use of descriptive terms about the artifact. For example, a system such as Tanagra [3] can create a large variety of platformer levels based on a series of spatial constraints over where platforms should be placed. However, these placements might not reflect what the user's intent might be in descriptive terms. For example, Tanagra cannot generate a level that is described as "suspenseful" or "frenzied" based on spatial constraints alone. This is especially important for non-technical users, as translating their intent to a series of spatial constraints that are expressed via graphical user interface control manipulations can become burdensome to the user.

We believe that natural language interfaces address some of these issues with mixed-initiative PCG systems by allowing aspiring non-technical users to describe a generated artifact in terms of expressive descriptive language. By mapping a series of natural language concepts to combinations of different parameters in the design space of the procedural generator, users can explore the design space in descriptive terms of the expected artifact instead of a series of logical, numerical, or spatial constraints. 
One example of such a system is CADI [4], a mixed-initiative automated game design tool for discovering Pong [5] variants. In CADI, users can use affective terms, expressed using natural language, to describe a variation of the game Pong. Internally, CADI maps each expressive term to a series of parameters and values in the design space of Pong variations and makes a move in the design space towards a solution that reflects these expressive terms. In this way, a designer is able to directly describe their expressive intent using natural language, thus reducing much of the difficulty inherent in parameter-driven design space exploration.

While the notion of implementing a natural language interface for a PCG system addresses the issues of reducing interface complexity and providing a model for designer intent, there are design concerns related to utilizing natural language as input for PCG systems. We identify three potential issues that can arise when implementing a natural language interface such as: (a) finding an acceptable initial solution in the design space that leads to a meaningful interaction with the system. (b) avoiding linear workflow issues when interacting with a natural language interface compared to the "freeform" affordances that graphical user interface controls allow, and (c) avoiding frustration with prolonged interactions with a natural language interface.

In this paper, we provide a detailed exploration of how a technical designer can approach the task of implementing a natural language interface for mixed-initiative PCG systems to leverage the advantages of natural language interfaces such as interface complexity reduction and designer intent modeling by exploring the following research questions:

- How do we define a design vocabulary for a generative system?

- How do we map concepts from a design vocabulary to a combination of controls such that the user can efficiently explore the design space?

- What conversational design patterns do we support in designing a natural language interface for PCG systems?

In the following sections address these questions by providing a methodology that describes how technical designers and researchers can create meaningful natural language interactions for PCG systems destined for a beginner non-technical user. In addition, we provide a case study of how this methodology can be implemented using an existing procedural tool inside the Unity3D engine, the Water4 plugin, finally a discussion of how the issues described above are addressed to create a natural language-based interface PCG system.

\section{Related Work}

The work described in this paper is informed by developments in natural language interfaces for design, existing mixed-initiative approaches to PCG, and theory concerning the design of generative design tools.

Several mixed-initiative PCG systems have addressed the problem of easing design space exploration. The typical approach provides a graphical user-interface which permits interaction and collaboration with the algorithmic back-end of the system, thereby supporting co-creation of a novel artifact. Notable systems in this area include Ropossum [7], Sentient Sketchbook [8], and Tanagra [3], which permit users to create levels for the game Cut the Rope, RTS games, and platformers respectively. Other developments in mixed-initiative PCG systems can be seen at the intersection of Automated Game Design (AGD) and mixed-initiative PCG. Some exemplary systems in this field are Cillr [1,2] and Wevva [9]. In Cillr and Wevva, users are provided with the tools to create small games on a mobile device.

Natural language inputs to design tools has been explored in the area of AI-assisted design. The AGD system ANGELINA-3 uses natural language input from the user in which meaning is extracted to create a series of aesthetic elements for generated games. Words are mapped to related concepts mined from the web, thereby creating necessary assets such as textures, music, and commentary for a generated Metroidvania style game. Other AGD systems that use a natural language input are Interactive Game-Design Assistant [10] and Game-o-Matic [11] which take a natural language representation of a series of concepts and relations, that are in turn are mapped to a series of game mechanics to generate Warioware Inc. [12] style microgames. Outside of game design and PCG, there are design systems that operate over natural language input in order to generate different types of artifacts, as well as constraints. For example, Cheong et al. $[14,15]$ convert natural language input into design constraints in mechanical CAD applications. Firedrop.ai's system Sacha [16] uses natural language input to generate websites, guiding the designer using a conversational interface.

Our work is informed by the concept of Casual Creators by Compton and Mateas [17], specifically their casual creator design patterns. We draw inspiration from design patterns such as "providing instant feedback", "avoiding a blank canvas initial solution", and "modifying meaningful parameters" within the design space. By applying these design principles to natural language interfaces in PCG, we can provide an experience that allows non-technical users to engage with generators that can be perceived as technically daunting.

Furthermore, we are informed by Colton et al.'s [18] parameter design methodology for casual creators. Their methodology provides a set of best practices on how to design a parameter space in the context of PCG tools, such as creating exemplars that capture special points of interest in the design space, the separation of parameters according to functionality, and the creation of "emergent features" by combining parameters that allow multi-dimensional design space movements. In addition, this methodology provides grounding on how to understand the design space of a generator from a parameter design perspective, compared to approaches that focus on the analysis of a generator's output.

\section{Methodology}

In order to understand how to implement a natural language interface for a PCG system, we must first address the interaction 
model inherent to such an interface. Compared to the traditional "freeform" nature of a graphical user interface containing a different control for each parameter in the design space, a natural language interface affords a more linear mode of interaction analogous to a conversation between the user and the procedural generator.

\subsection{Interaction model}

The interaction model that the user (e.g., a technical artist, or a gameplay programmer) of a natural language interface for a PCG system would use can be distilled to the five following steps (see Figure 1).

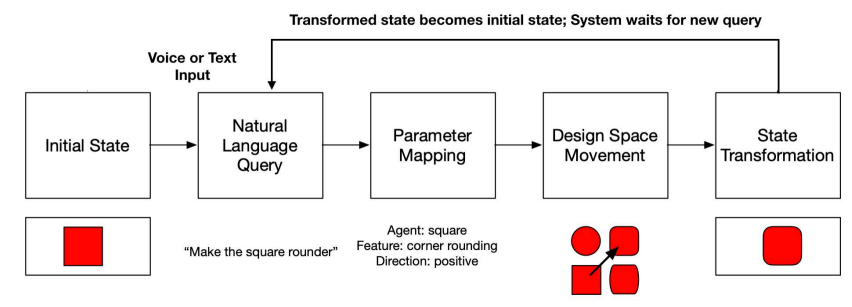

Figure 1: Architecture diagram for a natural-language interface for PCG Systems

1. Initial State: An initial point of reference is presented to the user to avoid the problem of starting with a blank canvas. Depending on the artifact to be generated, the designer of the system can choose an inspiring example or allow the user to choose an example from a series of reference points (e.g. sample output chosen by the tool's developer) in the design space.

2. Natural Language Query: The user inputs a natural query related to the artifact. This can be done via voice or text. For instance, a query can be in the form of: "make the artifact/subcomponent\} \{quantifier\} \{concept\}". The structure of accepted queries can be authored by the system's designer or used as unstructured text. This query is passed to a Natural Language Understanding (NLU) unit for interpretation.

3. Parameter Mapping: The NLU component interprets the natural language query and then maps it to a combination of features in the design space. For example, in the implementation of CADI [4], a concept such as "cold" maps to aesthetic features in the design space such as the color palette (bluer tones) and particle effects (trail rendering systems, particles on collision), whereas a concept such as "unfair" affects mechanical features in the design space such as the size of the paddles (scale of paddle in the game world) in the game. Additionally, the NLU component can understand the direction in the design space by means of a quantifier keyword such as "more" for a positive direction, and "less" for a negative direction.

4. Moving in the Design Space: Having extracted the affected features and the direction of movement in the design space, the system applies the transformation of the current solution and retrieves the new point in the design space. The magnitude of the jump in the design space can be either left to the user's desire by providing controls for the sensitivity of movement in the design space (e.g. controls for how much does a parameter change) or using assumptions made by the system designer (e.g. using a constant rate of change for certain parameter, setting upper and lower values to parameters).

5. New State Loop: The transformed solution is presented to the user, and it becomes the current state. Flow now loops back to \#2, as the system uses awaits a new natural language query.

Having described the interaction model for a natural language interface in PCG, we now detail the methodology that the designer of a PCG system would use for creating the NLU component, feature mapping, and design space movements. Figure 2 provides an overview of the methodology steps described in the subsections below.

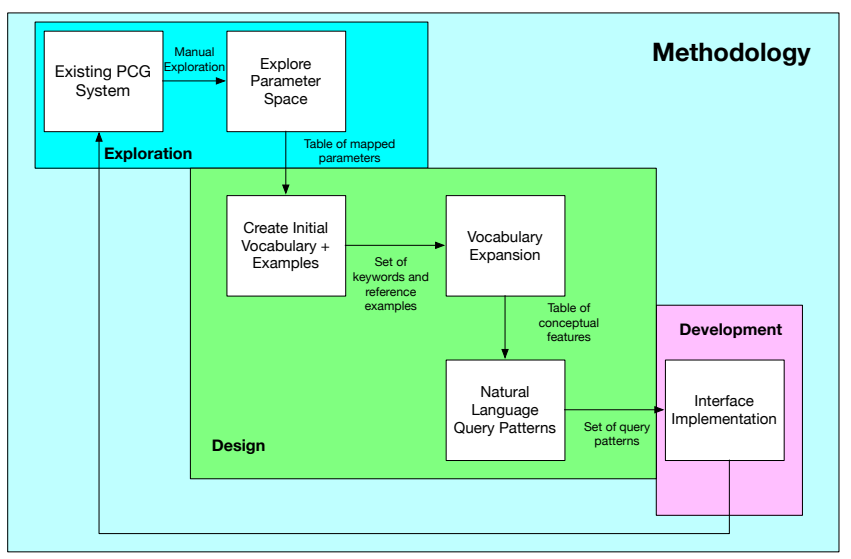

Figure 2: Process diagram for the methodology used to develop a natural language interface for PCG systems.

\subsection{Explore Parameter Space and Create Initial Vocabulary}

The first and most important step in the creation of a natural language interface for a PCG system is to understand the nature of the generated artifact by manually exploring the parameters of the procedurally generated design space, after which a series of natural language keywords can be applied to a combination of features in the design space.

When exploring the parameters of the design space, it is important to understand what is being generated in terms of the sub-components of the generated artifact. For example, in CADI [4], the subcomponents are the balls, paddles, and the arena for each Pong variation. After separating the generator into its functional sub-components, we now can explore and map the features that comprise them, such as aesthetics, and mechanics. Features that control colors or particle effects can be mapped as aesthetic characteristics, whereas physics parameters can be assigned to mechanical characteristics. This understanding of what aspect of the generator and what sub-component maps to 
what functionality of the generator can provide an initial ontology for the design vocabulary that can be generated.

Having mapped out the functional aspects of the generator into sub-components, a series of reference points based on natural language keywords can be made. These reference points can be seen as an initial mapping of our keywords in the design vocabulary to a specific point in the design space. This will comprise our initial design vocabulary. This is similar to the "capture an inspiring example" phase in Colton et al.'s [18] methodology. The first step in this phase is to find the desired keywords associated with the generated artifact. In the case of CADI [4], the basis for the design vocabulary was Swink's Game Feel theory [19] which focuses on the affective experience elicited by a game's design. This resulted in a design vocabulary, based on affective keywords, that could map to the generated artifact's control scheme, physics, and aesthetics. Some keywords associated with the initial design vocabulary for CADI were "cold", "unfair", and "sticky". In systems that deal with one specific type of artifact (such as asset generators) the initial design vocabulary can be defined using adjectives commonly applied to the represented artifact. One example would be to describe a tree with words such as "leafy", "twisted" or "blossoming", where as an ocean water generator could use words like "calm"," turbulent" or "tropical". In this phase, the end-users can be queried about how they would describe the generated artifacts by showing them random or manually selected reference points in the design space and asked to describe some of the qualitative characteristics of the artifact. This can be done via a user study with potential end-users of the system, or via crowdsourcing platforms.

With this initial set of descriptive keywords, the PCG system designer can now create a reference example in the design space that captures the meaning of the keyword. A reference example is a specific point in the design space that represents an archetypical artifact described by the keyword. While creating these examples it is important to record what variables influenced the design space exploration the most.

\subsection{Design Vocabulary Expansion and Conceptual Feature Design}

Now that an initial design vocabulary has been set for the generated artifacts, we can expand the vocabulary to provide more options for expressing desired intent about the artifact. One of the simplest ways to expand the vocabulary is synonyms of the initial keywords in our initial design vocabulary. Synonyms allow the system's designer to enhance the NLU component's capabilities to detect intent for similar concepts, as the mental model of what language to use to describe an artifact may vary from user to user. By grouping similar words to one single concept, the designer can cover many similar use cases.

Another technique is to create a counter-example for each initial keyword. This approach uses the same features, but with values mapped to the antonym of the original keyword. This technique allows for the creation of a continuous design axis. A benefit of this is it allows the user to explore a large combination space of desired feature combinations without having to tweak one parameter at a time. In addition, synonyms can be added to the counter-example as an expansion of the concept. One such example can be seen in CADI [4], where the words "warm" and "cool" form a single natural language concept that modifies the color palette between blue and red hues. In this case, the "cool" example was defined in the initial exploration of the design space, upon which a counter-example ("warm") was worked using the same set of modified features. In addition, synonyms were used (e.g "cold", "frigid", "icy", "hot", "fiery", "blazing") to expand the design vocabulary in order to allow more options for the user to express their intent.

Tying both the design vocabulary generation and expansion we arrive at a series of mappings of natural language keywords to a set of compound core features for our generator. We refer to these mappings as conceptual features in our natural language interface design. These conceptual features conform the core concept for using natural language in PCG systems as they capture different intents from the system's users.

\subsection{Natural Language Query Patterns}

Having defined a set of conceptual features representing the combinations of features that describe an artifact using expressive language, the next step is the development of patterns for user queries through the natural language interface. These queries are what allow users to perform actions on the design using the conceptual features that were defined in the previous phase.

One initial strategy is to form a series of patterns in the form of a declaration about the artifact and its identified subcomponents using the conceptual features. These patterns take the following form:

1. A noun referring to the artifact or a sub-component: In CADI [4] it could be the entire game, the paddles, or the Pong ball.

2. A word that signifies the direction of movement along the axis of a conceptual feature: Words like "more" or "less" are good starting points to signify a positive or negative movement in the design space. We call these words quantifiers and address their handling later in this subsection.

3. Finally, a conceptual feature to modify in the design space.

Following this rule, we end up with queries such as "make the artifact/sub-component more/less conceptual feature" which provides the user with a set of initial exploration actions. While this pattern allows for a "quick and dirty" approach to moving in the design space it does not allow for more fine-grained control of design space exploration. This is because this pattern relies on default assumptions set by the system's designer about how much or little the quantifier moves in the design space.

A different approach to learning what kind of natural language queries might arise when interacting with the PCG system is to have potential end users of the system to participate in a "Wizard of Oz" type user study. In this study, the users are asked by the researcher or technical designer to interact with an "idealized" version of the natural language-based interface. This can be either the researcher manually manipulating the PCG system behind the scenes, or by a paper prototype in which the 
A Methodology for Designing Natural Language Interfaces for Procedural Content Generation

researcher or designer shows the changes to the artifact manually on the prototype. This type of approach while time consuming, might enable the researcher or technical designer understand what kinds of emergent queries outside of their mental knowledge might occur when interacting with the tool. In addition, this approach might also help enrich the vocabulary as new words outside of what has been authored up to this point can be added based on user feedback.

We would suggest that a hybrid approach to this task might yield the best results as the queries designed by the researcher or technical designer get to be tested in the user study, while learning new emergent queries that potential users of the system might use when interacting with our natural language-based interface.

Now that we have an initial set of actions to operate in the design space using expressive language, we can expand our patterns to handle more specific cases. This is done by using verbs to map the natural language query to specific functionalities in the generator. For example, the phrases "look like" affect visual parameters in the design space, while phrases like "move like" affect motion components in our generator. In addition, we can constrict the set of conceptual features that map to each verb, given that a mapping of conceptual features to functionalities has been explored in previous phases of the design of the natural language interface.

Having established some initial patterns defining general actions and functionality-specific actions, we address the need for two specific use cases related to more direct control of the generator. One is numerical control of our conceptual features, and the other is direct manipulation of the parameter space in numerical terms. Our first pattern is to handle cases in which a user can say "Make the artifact/sub-component X\% more/less conceptual feature". In this case, we want to keep track of where in the design space the user is located to apply a transformation over the values affected in the conceptual feature understood from the query. Our second pattern is to allow direct control of the feature space to the user. This can be done by simply mapping named keywords for each feature in the design space to allow a straightforward manipulation of the parameter space for more advanced users that need fine-grained control to fine tune their expected artifacts. However, this runs counter to our goal of having conceptual terms which map across multiple controls at the same time.

Finally, we address the concept of quantifiers, which are keywords that represent the direction of movement in the design space for a conceptual keyword. We mentioned the usage of the keywords "more" and "less" as signifiers of positive and negative movement in the design space of a conceptual feature. To control this movement, we develop an approach for translating keywords into a numerical "step" movement in the design space, as described in Equation 1. In this equation, the granularity parameter controls the amount of movement desired in each "step". The quantifier $q$ (positive or negative) indicates the desired direction of movement. A magnifier parameter $m$ accounts for
FDG'19, August, 2019, San Luis Obispo, California USA

more dramatic jumps in the design space, which is mapped to phrases such as "even more" or "way less".

$$
\begin{gathered}
\text { step }=\mathrm{m}\left(\mathrm{q} * \frac{\mid \mathrm{max}(\text { param })-\min (\text { param }) \mid}{\text { granularity }}\right) \\
\mathrm{q}=\left\{\begin{array}{c}
1 \text { if quantifier }=\text { "positive" } \\
-1 \text { if quantifier }=\text { "negative" }
\end{array}\right. \\
\mathrm{m}=\left\{\begin{array}{c}
1 \text { if not activated } \\
\mathrm{m} \in \mathbb{Z}: \mathrm{m} \in[2,10] \text { if activated }
\end{array}\right.
\end{gathered}
$$

\section{Equation 1: Step distance for relative movement in design} space

The user is typically presented with the option of setting the amount of granularity and the value of the magnifier parameter via two graphical user interface controls. This allows the user to determine and fine-tune the amount of change to best reflect their idea of the magnitude of movement in the design space. Alternatively, the designer can make assumptions of how much movement is expected from the model and further reduce interface complexity for the user.

\subsection{Addressing Design Concerns}

In summary, our methodology for creating a natural language representation of a PCG system design space begins from an initial exploration of the design space, proceeds to the development of a design vocabulary and conceptual features, and ends with development of the patterns for the natural language queries that are used to represent actions in the PCG system. We now address the emergent design issues that arise from such development, including words outside the design vocabulary and avoiding "junk" output.

While our design vocabulary and conceptual features might be able to capture the users' intent to a certain degree, expressive language queries can contain concepts that are outside the set of conceptual features. To avoid problems with a lack of feedback due to unrecognized keywords, a fallback strategy is needed. One such strategy is to use web data to define a default set of features. One example of this style of fallback strategy was implemented in CADI [4], where unrecognized color keywords led to a call to a RESTful API from colourlovers.com [19], which returned a color matching the keyword. By using this strategy, designers can have a larger set of keyword coverage beyond their defined design vocabulary and conceptual feature set without sacrificing feedback for users when interacting with the system.

Another issue is finding regions of "junk" output. This can be defined as regions in the design space of the generated artifact that are not consistent, present erratic behaviors, or are considered to be aesthetically unpleasant. This can be addressed during the explorative phase of the methodology by capturing what are considered "acceptable" values by setting upper and lower bound values for each explored parameter. While this strategy might reduce the number of solutions in the design space that can be 
explored, avoiding "junk" output can help achieve a sense of consistency in our natural language-based interface system.

\section{Using the Methodology for a Water Simulation}

Having described our methodology for creating a natural language-based interface for PCG, we now show an example implementation made for an existing plug-in in the Unity3D game engine called Water4. Water4 is a shader-based plugin for the Unity3D engine that creates volumes of water and uses a Gerstner-based wave simulation to animate the water mesh it generates.

\subsection{Design Space Exploration and Initial Design Vocabulary}

We followed the methodology presented above to generate the conceptual features and natural language-based queries for interaction with Water4. The first step was to understand the generative design space of the plug-in and to separate the subcomponents and parameters based on their functionality. This was done by a manually exploring the design space of the plug-in, modifying one parameter at a time. In this case we identified the following sub-components in the generated artifact: a water mesh, a shader for the water, a shader for foam, and a Gerstner displacement-based simulation for the waves. Upon investigation of the plug-in's parameters we identified 46 different modifiable parameters: 18 for modifying the water mesh and foam, and 24 for modifying the wave simulation. Figure 3 shows the mapping between parameters and functionality in Water4.

\begin{tabular}{|c|c|c|}
\hline Component & Parameters & Functionality \\
\hline Water Mesh & $\begin{array}{c}\text { Tiling } 1 / 2 \text { (Vector } \times \text { 2) } \\
\text { Direction 1/2 (Vector2 x2) }\end{array}$ & Visual \\
& Refraction Color & Visual \\
& Reflection Color & \\
& Texture & \\
& Normals (float $\times 3)$ & \\
& Fresnel (float x3) & Visual \\
& Fading (float x3) & \\
\hline Foam Shader & Foam Texture & Motion \\
& Foam Intensity (float) & \\
\hline Wave & Foam Cutoff (float) & \\
Simulation & Gerstner Amplitude (Vector4) & \\
& Gerstner Frequency (Vector4) & Gerstner Steepness \\
& (Vector4) & \\
& Direction Scale 1/2 (Vector4 x2) & \\
&
\end{tabular}

Figure 3: Mapping the parameter space in Water4 by functionality and subcomponent

Now that our parameters space has been mapped out by functionality and artifact sub-components, we establish our initial design vocabulary. This was accomplished by manually creating examples that represented different aspects of the artifact, such as different color palettes and wave behaviors. Sets of keywords that described the ocean, waves, and water were also recorded. Some examples of the initial design vocabulary were words describing the ocean such as "turbulent", "calm", and "Caribbean".

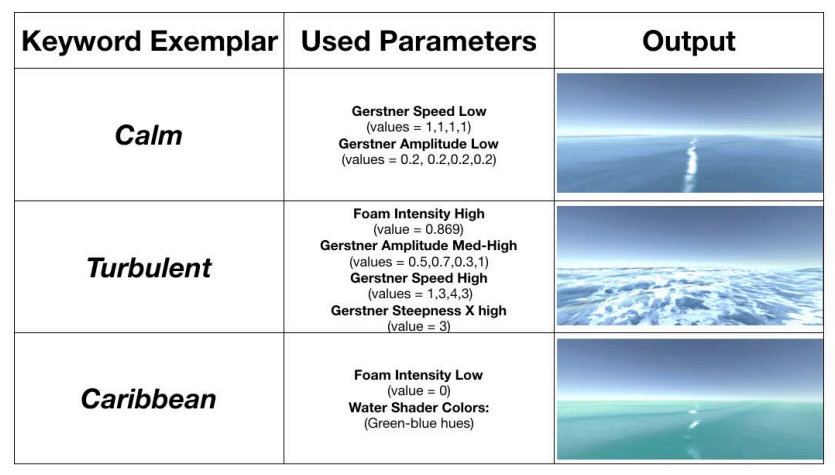

Figure 4: Initial design space examples in Water4 with associated parameter values.

This led to the creation of 10 initial examples mapped to a keyword, with associated parameters recorded. In this case the word "Caribbean" maps mostly to the color of the water rather than the shape of the waves, whereas examples like "calm" and "turbulent" are mostly related to the behavior of the wave simulation. Figure 4 shows some exemplary artifacts in Water4 for the keywords used in this paragraph.

\subsection{Design Vocabulary Expansion and Conceptual Feature Design}

Starting from these examples, we expanded the design vocabulary by using synonyms to create additional positive instances of the conceptual feature. For example, when designing the "calm" example we noticed that the wave simulation parameters comprised the set of modified features for our generated artifact's wave simulation subcomponent. With this in mind, we generated a counterexample for "angry" using the same features in order to create a continuous design axis that allows the user to move in the design space between "angry" and "calm" while using the same set of generator features. Figure 5 shows an illustration of the design axis generated for "calm" and "angry" in Water4.

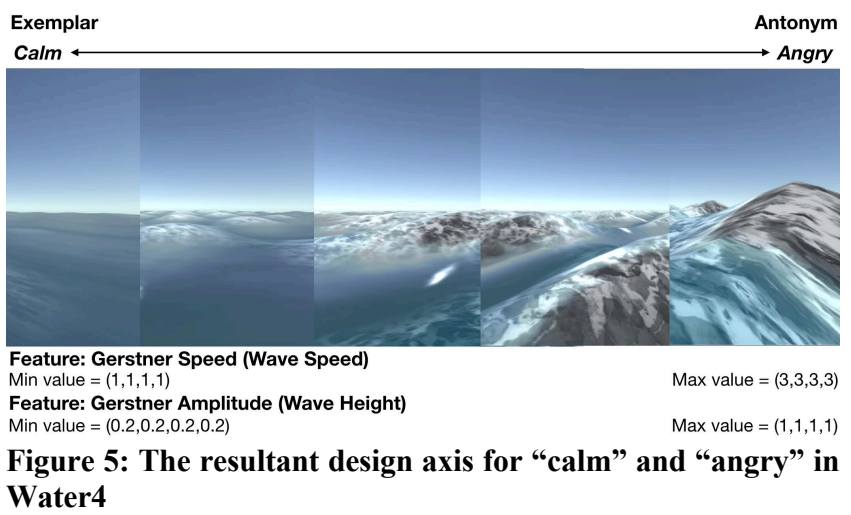


Following this example, we proceeded to develop each keyword of our initial design vocabulary by using synonyms and antonyms to create our conceptual feature set. Figure 6 shows a sample of our expanded conceptual features for the examples in Figure 4.

\begin{tabular}{|c|c|c|}
\hline $\begin{array}{c}\text { Conceptual } \\
\text { Feature Name }\end{array}$ & $\begin{array}{c}\text { Word Concepts (Positive } \\
\text { and Negative) }\end{array}$ & $\begin{array}{c}\text { Parameter } \\
\text { Combinations }\end{array}$ \\
\hline Calm-Angry & Calm, tranquil, peaceful & Gerstner Speed \\
& Angry, tempestuous, wild & $\begin{array}{c}\text { Gerstner } \\
\text { Amplitude }\end{array}$ \\
\hline Clear-Turbulent & Clear, clean, pristine \\
& Turbulent, murky, foamy & $\begin{array}{c}\text { Foam Intensity } \\
\text { Foam Cutoff }\end{array}$ \\
\hline WaterTemperature & Caribbean, tropical, warm & $\begin{array}{c}\text { Water Shader } \\
\text { Colors (green-blue } \\
\text { to dark-blue) }\end{array}$ \\
\hline
\end{tabular}

Figure 6: Sample conceptual features used in our Water4 natural language interface.

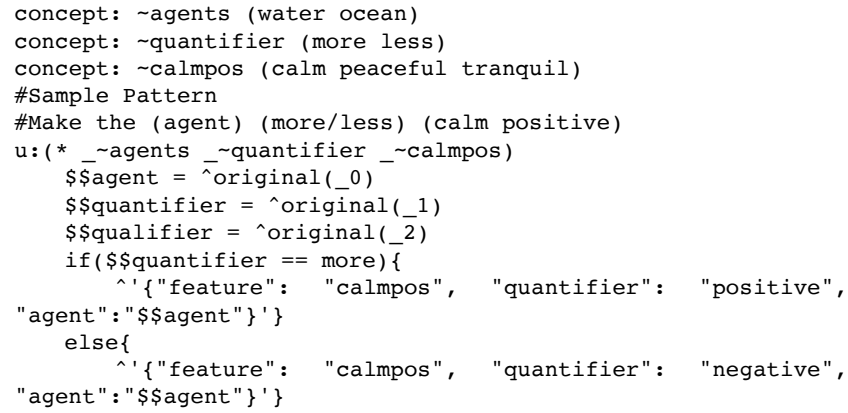

Figure 7: Sample Chatscript implementation of natural language query patterns.

\subsection{Natural Language Query Patterns}

Following the development of our conceptual feature set, we proceeded to build the NLU component in Chatscript [20]. The way Chatscript operates is by matching authored patterns for natural language queries. These patterns are a series of keywords that are defined in our NLU component that are arranged in a specific order. In this component we added the definitions for our design vocabulary grouped by conceptual features, the query patterns for controlling conceptual features, and query patterns for direct control of the parameter space in Water4. Figure 7 shows an example of a query pattern and a definition for a concept using keywords in our design vocabulary.

\subsection{Implementation}

After developing our NLU component in Chatscript, we developed the following components in Unity3D following the architecture in Figure 8: A parameter modification script, an implementation of the quantifier model and a connection script to Lahar [22], a REST API that handles the Chatscript connection for our NLU component. Finally, we developed the graphical user interface that allows natural language-based interaction for Water4. In this step, we added controls for voice recognition, textual input, controls for the manipulation of the quantifier model (granularity and magnification), and an option to save their final artifact such that it can be loaded into other Unity3D projects. With this, we ended up with the final implementation of our system as evidenced in Figure 9.

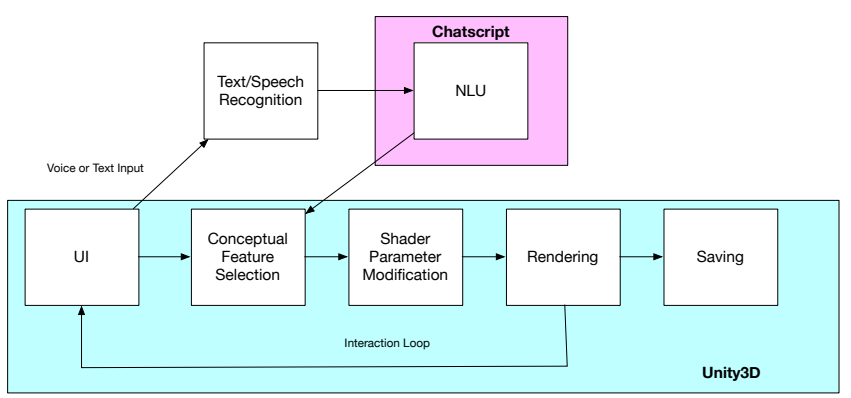

Figure 8: Architecture Diagram for our Water4 example implementation

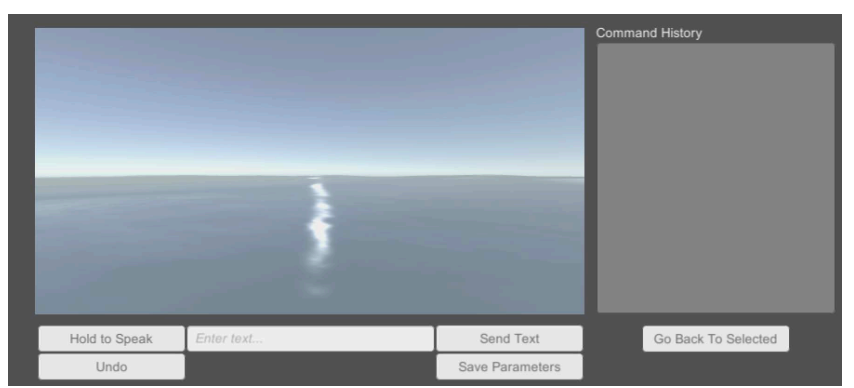

Figure 9: Screenshot of the implemented UI for our natural language interface for Water4.

\subsection{Addressing Design Issues}

We now address the issues discussed in the introduction concerning blank canvas issues, interaction attrition, and linear workflow issues. To address our blank canvas issue, we set the initial state of the generator to a default value provided by the Water4 designers. This avoids the problem of starting at an extreme point in the design space (such as all parameters set to zero). By presenting the user with an internally consistent default configuration they are able make an initial query that takes them closer to their desired solution in the design space.

We address the interaction attrition issue by allowing the user to trace their steps back in the design space and by showing their history of interactions. In this way they can easily correct undesired moves in the design space without having to restart from an initial default state. We believe that keeping track of states and providing mechanisms to move within the interaction history allows the designer to efficiently explore the design space as they can explore different pathways without having to start over again every time the output is undesired. 
We address linear workflow issues by allowing direct manipulation of the parameters in Water4 using natural language queries. This is seen as a fallback strategy analogous to direct modification of the plug-in. This type of interaction allows for a more freeform style exploration in conjunction with our implementation of design space exploration history. Direct parameter manipulation allows the designer to freely move between previously visited points and use fine-grained controls to explore the design space without having to recur to expressive language as their only interaction option.

\subsection{Example Interaction Session}

Finally, we provide an example session of how a designer can interact with our user interface in accordance with the interaction model shown in Figure 1. In Figure 10 we show how a user can interact with our systems interface by using natural language queries, and the internal steps in our pipeline as the user moves through the design space of Water4, such as what conceptual features are chosen in the NLU component, and the associated feature transformations and the output shown to the user.

\begin{tabular}{|c|c|c|c|c|}
\hline State & $\begin{array}{c}\text { Natural Language } \\
\text { Query }\end{array}$ & $\begin{array}{c}\text { Conceptual } \\
\text { Feature }\end{array}$ & $\begin{array}{c}\text { Feature } \\
\text { Transformation }\end{array}$ & Output \\
\hline Initial & none & none & none & \\
\hline Solution 1 & $\begin{array}{c}\text { "make the water more } \\
\text { stormy" }\end{array}$ & Calm-angry & $\begin{array}{c}\text { feature: GerstnerSpeed, } \\
\text { GerstnerAplitude } \\
\text { quantifier: positive }\end{array}$ & $\begin{array}{c}\text { feature: } \\
\text { GerstnerSteepness } \\
\text { quantifier: positive }\end{array}$ \\
\hline Solution 2 & "make the waves taller" & Height & $\begin{array}{c}\text { feature: Foamintensity, } \\
\text { FoamCutoff } \\
\text { quantifier: negative }\end{array}$ & \\
\hline Solution 3 & $\begin{array}{c}\text { "make the foam less } \\
\text { turbulent" }\end{array}$ & Clear-Turbulent & feature:ShaderColor \\
quantifier: negative
\end{tabular}

Figure 10: Example session showing natural language interaction with Water4.

\section{Conclusions and Future Work}

In this paper we introduced and discussed a methodology to allow PCG systems designers to allow natural language input in their systems. This methodology follows a series of steps that allows a mapping of parameters in the design space of the PCG system to a series of keywords that are descriptive of the generative artifact and which allow the user to express design space movements in natural language form. This is accomplished by: (a) an initial exploration of the design space to understand the functionality of each parameter, (b) the creation of a design vocabulary, (c) a set of descriptive keywords related to the artifact's characteristics and a set of example design points related to each word in the design vocabulary, followed by (d) an expansion of the design vocabulary through the usage of synonyms and counter-examples that will form a conceptual feature. Afterwards, we design a series of natural language query patterns that map actions in our generator to keywords and conceptual features. Finally, we discuss the implementation of a model to translate our natural language mappings to numerical movements in the design space. In addition, we provide an example implementation of our methodology to an existing plug-in in Unity3D called Water4.

We believe that using natural language-based interfaces provides designers a way to let less technically oriented users explore the design space of a PCG system. By allowing the usage of expressive language, users can explore the design space of a PCG system in terms of the desired characteristics of the artifact, rather than a direct freeform manipulation of the parameters that compose the design space of the system. The affordances allowed by natural language descriptions of the artifact and the usage of conceptual features also allow for a reduction of interface complexity of mixed-initiative PCG systems. We believe that this methodology and its applications can improve the user experience of PCG systems, as well as providing a further reduction of the technical barrier of usage for systems.

Further directions in this work include the development of a unified architecture and knowledge representation for handling the creation of conceptual features and natural language queries. For instance, the creation of a framework for an editor-based plug-in that allows a connection to a PCG system in Unity3D using natural language input can help PCG system designers reach a wider user base such that less technical users can embrace the expressive power of procedural generation without a high technical barrier of entry.

\section{REFERENCES}

[1] M.J. Nelson, S.E. Gaudl, S.Colton, et al. 2016. MixedInitiative Approaches to On-Device Mobile Game Design. Proceedings of the Mixed Initiative Creative Interfaces workshop at CHI 2016.

[2] M.J. Nelson, S.E. Gaudl, S.Colton, et al. 2017. Fluidic Games in Cultural Contexts. Proceedings of the Eighth International Conference on Computational Creativity.

[3] G. Smith, J. Whitehead, M. Mateas. 2010. Tanagra: A Mixedinitiative Level Design Tool. In Proceedings of the Fifth International Conference on the Foundations of Digital Games.

[4] A. Mobramaein, M. Behrooz, J. Whitehead. 2018. CADI: A Conversational Assistive Design Interface for Discovering Pong Variants. Proceedings. Artificial Intelligence in Interactive and Digital Conference 18, AAAI

[5] A. Alcorn 1972. Pong (Game). Atari 2600.

[6] A. Mobramaein, J. Whitehead, C. Chakraborttii. 2018. "Talk to Me About Pong: On Using Conversational Interfaces for Mixed- Initiative Game Design." AAAI Spring Symposium Series The UX of AI.

[7] N. Shaker, M. Shaker, J, Togelius 2013. Ropossum: An Authoring Tool for Designing, Optimizing and Solving Cut the Rope Levels. Proceedings. Artificial Intelligence in Interactive and Digital Conference 2013.

[8] A. Liapis, G. Yannakakis, J. Togelius. 2013. Sentient Sketchbook: Computer-Aided Game Level Authoring. Proceedings of the Eighth International Conference on the Foundations of Digital Games. 
[9] E.J. Powley, M.J. Nelson, S. Gaudl et al. 2017 Wevva: Democratizing Game Design.

[10] M.J. Nelson, M. Mateas. 2008. An Interactive Game-Design Assistant. Proceedings of the 13th International Conference on Intelligent User Interfaces

[11] M. Treanor, B. Blackford, M. Mateas, I. Bogost. 2012. Game-O-Matic: Generating Videogames That Represent Ideas. Proceedings of the The Third Workshop on Procedural Content Generation in Games, 11.

[12] K. Takeuchi, G. Abe, R. Takahashi. 2003. WarioWare Inc.: Mega Microgames! (Game), Gameboy Advance:

[13] Cook M., Colton S., Pease A. 2012. Aesthetic Considerations for Automated Platformer Design. In Proceedings, The Eighth AAAI Conference on Artificial Intelligence and Interactive Digital Entertainment,

[14] H. Cheong, W. Li, L.H. Shu, et al. 2014. Natural Language Problem Definition for Computer-Aided Mechanical Design. Proceedings of the DSLI Workshop SIGCHI.

[15] H. Cheong, W. Li, F. Iorio 2016. Automated Extraction Of System Structure Knowledge From Text. Proceedings of the ASME 2016 International Design Engineering Technical Conferences \& Computers and Information in Engineering Conference IDETC/CIE 2016.

[16] Firedrop Ventures. 2018, sacha.ai, https://sacha.ai

[17] K. Compton, M. Mateas. 2015. Casual Creators. In Proceedings of the Sixth International Conference on Computational Creativity.

[18] Colton S, et al.; A Parameter-Space Design Methodology for Casual Creators. 2018, http://repository.falmouth.ac.uk/2893/.

[19] Colourlovers.com, Colourlovers API

[20] B. Wilcox. 2010 Chatscript http://chatscript.sourceforge.net/

[21] Google Inc; Google Speech API

[22] S. Cousins. 2015, Lahar 